\title{
Communication via warm haptic interfaces does not increase social warmth
}

\author{
Christian J. A. M. Willemse ${ }^{1,2}$. Dirk K. J. Heylen ${ }^{1}$. Jan B. F. van Erp ${ }^{1,2}$
}

Received: 5 July 2017 / Accepted: 21 August 2018 / Published online: 9 October 2018

(c) The Author(s) 2018

\begin{abstract}
Affective haptic interfaces are designed to influence one's emotional and physiological state via the sense of touch, and may be applied as communication media to increase the sense of closeness. Recent research suggests that stimulation with physical warmth can enhance this interpersonal closeness: a physical-social warmth link. It is often suggested that this link may be particularly suitable for application in affective haptic interfaces, but the scientific evidence is inconclusive. In this work we investigated whether adding physical warmth to a communication medium — an interactive teddy bear — could increase social connectedness between remotely located interactants and could provide physiological comfort during stressful circumstances. Moreover, we investigated whether the warmth could best be presented to the users as a mere physical attribute of the medium or as mediated body heat; thereby manipulating the attribution of the warmth to either a non-social or social source. The results of two studies in which participants ostensibly received a message from an unknown other (Study $1, N=65$ ) or comforting messages from their own partner (Study 2, $N=62$ ), and meta-analyses did not provide support for the hypotheses that warmth, purely physical or attributed to one's partner, can positively influence one's social and physiological state. Although future research should corroborate our findings, they indicate that the physical-social warmth link may not be as applicable in affective mediated communication as suggested.
\end{abstract}

Keywords Affective haptics · Computer mediated communication · Physical warmth · Social warmth · Attribution

\section{Introduction}

Over the years, a plethora of computer mediated communication (CMC) devices and applications has been developed to address humans' innate need for social connectedness and intimacy [8]. However, CMC is often criticized for impoverishing and devaluing human communication [91], and may even have contributed to the prevalence of individualism and feelings of loneliness [15]. That is to say, brief textual or audio-visual messages supposedly cannot match the intimacy as experienced during face-to-face contact. To achieve more intimate $\mathrm{CMC}$, interfaces that modulate one's emotions via the sense of touch [32] could be particularly suitable. These 'Affective Haptic Interfaces' [90]

Christian J. A. M. Willemse

c.j.a.m.willemse@gmail.com

1 University of Twente, Human Media Interaction, P.O. Box 217, 7500 AE Enschede, The Netherlands

2 TNO, Perceptual and Cognitive Systems, P.O. Box 23, 3769 ZG Soesterberg, The Netherlands could be utilized in a non-social way, by merely drawing upon their physical qualities. Different materials and textures (e.g., [35,81,84,96,106]), or haptic actuators such as vibrotactile [79,80], air jet based [89], friction-based [77], or force feedback [5] can be employed to elicit affective responses in the receiver. Another approach to use affective haptics is to directly attribute the physical stimuli to a social source, as is for instance the case with touching social robots [88,101] or in Mediated Social Touch [26,36,40]. The latter approach may alter the perceptions of the physical stimuli and the associated affective responses due to the social connotations the message entails. To better understand how affective haptic CMC that is aimed at increasing social connectedness and intimacy should be designed, it is important to discern between the non-social and social qualities of physical stimuli.

Using non-noxious physical warmth is often proposed for affective CMC $[9,33,47,87,98]$, as it provides a pleasant, comfortable feeling, is semantically associated with positive emotional valence [9], and activates brain regions associated with positive emotions [82,104]. Moreover, perceptions of 
physical warmth can activate perceptions of 'social warmth' (i.e., the experience of being connected to another) $[47,102]$ : a physical-social warmth link. In practice this means that holding warm objects, or residing in relatively warm environments, may activate pro-social behaviors and cognitions [44] and decrease feelings of loneliness [6]. While warmth may elicit beneficial responses in every user, it may be particularly suitable for people that suffer from loneliness, stress, or psychological pathologies [6,72]. In this work, we set out to test the hypothesis that the physical-social warmth link is also applicable in CMC, and that physical warmth thus can increase feelings of connectedness and comfort between remotely located interactants. Moreover, we investigate whether attributing different meanings to the warmth results in different affective responses from the receiver. That is, the warmth is described to the participant either as a mere physical, non-social quality of the interface, or as (social) warmth that represents the sender's body heat.

In the remainder of this paper we will substantiate our hypotheses by discussing why physical warmth actually may be valuable for social connectedness and comfort in CMC. Moreover, we describe related work, and elaborate on the role of attribution in CMC, after which we describe and discuss two studies. With the work as presented here we intend to further inform the design of haptic CMC interfaces that can effectively provide feelings of social connectedness and comfort.

\subsection{The link between physical and social warmth}

The physical-social warmth link supposedly stems from our period as newborns, during which we are often held closely by our parents and perceive their bodily warmth [56,103], and during which the foundations of psychological mechanisms such as affect and social bonding are formed [11,44]. Moreover, the perceptions of physical and social warmth seem to share neurobiological mechanisms, which appear to be unrelated to the hedonic qualities of warmth [48]. Both the perception of physical and social warmth can activate the same areas in the insular cortex (responsible for social cognitions and emotions) $[19,47]$, and neurochemical substrates [48].

Perceiving physical warmth thus seems to imply physical closeness to a source of affiliation and therefore a sense of social connectedness. This suggestion was supported by a study in which people perceived the physical distance to a warm drink as smaller than to a cold drink, after holding it [28]. Related studies demonstrated that people, when residing in a warmer environment or when being primed with a warm object, use more language that is associated with feeling socially connected to others. Moreover, people focus more on relational characteristics of abstract figures rather than on their individual attributes, and they feel more social proximity towards another [45,78]. Also, the desire to bond was higher when primed with physical warmth [28]. This increased social connectedness resulted for instance in increased altruistic behavior such as sharing small gifts [44,102], whereas coldness made people invest less with an anonymous partner and decreased trust [53]. Moreover, holding a warm drink, contrary to a cold one, led people to perceive others as socially warmer [102]. They attributed more 'socially good' personality traits such as friendliness $[4,31]$ to an ambiguously described other person, whereas traits that are unrelated to this socially warm-cold dimension remained unaffected by the warmth.

The physical-social warmth link appears to be bidirectional. When people think about relationships and communal feelings [83], or when they are physically close to others [46], they tend to perceive the ambient temperature as higher. When people experience social stress such as exclusion or guilt, they perceive the temperatures as lower $[6,108]$ and they actually become physically colder as their skin temperature decreases $[41,49]$. Socially stressed people show an increased need for compensatory or restorative physical warmth. They for instance have more warm drinks or take more warm baths, and this warmth supposedly reduces the stress [6]. Since warmth may be beneficial for social connectedness, physical comfort, and psychological comfort, it seems suitable for affective CMC.

The exact underlying mechanisms of the physical-social warmth link are not fully understood though, as the research is still in its infancy. Moreover, the research may suffer from publication bias or even false positives, for instance due to underpowered studies [43]. This is reflected in failed attempts, with significantly larger sample sizes, to replicate the effects of warmth on perceived personality [63], the relation between communal feelings and the ambient temperature [25], and the need for compensatory warmth during social stress [24]. Since the majority of the effects of warmth has been found in direct contrast with coldness, and because coldness decreased altruistic behavior [53], it could be that physical coldness has a negative impact on people, rather than warmth having a positive effect [6,99]. Moreover, rather than being a specific response to social stress, the decrease in skin temperature may also be a common sympathetic response to negative, stressful events [54,75], which is reflected by vasoconstriction in for instance the fingertips or the tip of the nose. The inconclusiveness regarding earlier research on the physical-social warmth link and its underlying mechanisms warrants an empirical validation of the suggested beneficial effects concerning social connectedness and comfort of warmth in CMC $[9,33,47,72,87,98]$. 


\subsection{Warmth in computer mediated communication}

On the premise that social touch is one of the most intimate forms of face-to-face communication and is essential for wellbeing, social bonding, and behavior [29,30,32,66], affective haptics have been employed to simulate social touches with the intention to induce similar beneficial effects $[26,36,40]$. Since body warmth is an important physical quality of a human touch $[1,38]$, it has also been included in several mediated touch devices. Examples include artificial representations of hugging (e.g., $[22,86]$ ) and holding hands [34], and a more abstract interaction in which squeezing a "White Stone" results in the heating of another augmented stone [87]. Although such devices supposedly can enhance the social connectedness while being separated, and may be particularly suitable for the conveyance of affective messages [60,61], empirical evidence for this claim is sparse and inconclusive. Perceiving a warm "thermal hug" around the waist, that was sent by the partner during a mediated collaborative task, increased social presence [33]. Also, physical interactions with warm non-human social entities, robots with 'body heat', resulted in a more positive attitude towards these robots and a stronger sense of trust and friendship [67,69]. With regard to one's emotional state, research has demonstrated that stimulation with different thermal patterns elicits various emotional responses [76]. When haptic patterns consisting of warmth, vibration, and a tickle sensation were attributed to another person and presented while watching an emotional movie, these mediated touches reduced physiological stress responses with a similar rate as real human touch [13]. On the contrary, warmth did not affect the social perceptions of a fictitious peer after mediated interaction [99]. Applying the physical-social warmth link as means of affective CMC thus is not trivial. Moreover, it remains unclear whether the effects found were mainly caused by the attribution of the warm message to "the sender" and the associated social meaning, or merely by the physical qualities of the interface. In the former explanation, the warm messages could be considered a form of disclosing affect and personal emotional information; mechanisms that are known to contribute to intimacy during communication [16,58]. In the latter case, the effects of the warm messages would mainly draw upon the inherent physical comfort and/or the physical-social warmth link.

In the work as presented here, we intend to shed additional light on the roles that these aspects may have in affective haptic CMC, by actively manipulating both the physical temperature and the source, and thus the social meaning, of the warmth. This is done in two studies in which participants were invited to communicate with another person via an affective haptic communication interface, and to evaluate this haptic message afterwards. The interface contained heat-pads that were either switched off (i.e., the "Room Temperature" condition), or switched on. When the interface was warm, we manipulated the attribution of the warmth to either a non-social or social source. The warmth was either presented as a physical attribute of the interface that was switched on beforehand, and thus did not have social meaning (the "Artificial Heat" condition), or as a real-time display of the communication partner's "Body Heat", with inherent social meaning. During these two three-conditions between-subjects experiments, we set out to find support for the following hypotheses:

H1: Direct physical contact with warmth provided by a haptic communication medium will, contrary to neutral temperature, result in increased social connectedness, socially warmer perceptions of the other, and comfort.

H2: The beneficial effects of physical warmth are larger when the warmth is attributed to the other person (as mediated body heat), rather than to a non-social inanimate attribute of the haptic communication device.

With this work, we intend to further inform the design of affective haptic CMC interfaces by investigating the applicability of the physical-social warmth link. Moreover, we intend to further inform the approach to take in the design of effective affective haptic interfaces; for instance with regard to increasing social connectedness. That is, this work could show whether an approach that mainly relies on the perception of the physical qualities of the interface may already suffice in sorting beneficial psychological and physiological effects, or that more elaborate processing, such as interpreting the actual meaning or intentions of the haptic signal, is required.

\section{Study 1: haptic communication with an unknown other}

In the first study, participants were invited to engage in communication with an unacquainted communication partner, which in fact, and unknown to the participants, was fictitious. They were led to believe that the other person could see the participant and could send a message via a haptic interface, after which the participant was asked to assess this other person based on the received message. The main aim of this study was to gain insights in the general applicability of the physical-social warmth link in CMC.

\subsection{Methods}

\subsubsection{Participants}

A total of 65 participants took part in the study and were randomly assigned to the Room Temperature $(n=22 ; 15$ male, 7 female), Body Heat ( $n=22 ; 10$ male, 12 female), 
or to the Artificial Heat ( $n=21 ; 15$ male, 6 female). The mean age of the mostly student participants was 21.48 ( $S D$ : 2.03, range 18-27) and 25 participants (38.5\%) were female. Participants did not receive compensation for their participation. This study was reviewed by the internal review board and carried out in accordance with the Helsinki Declaration of 1975, as revised in 2013 [105], and participants gave written informed consent prior to the experiment.

\subsubsection{Setting and apparatus}

Participants communicated with the fictitious other person via the haptic interface "Nakama" [100], which is an actuated teddy bear (see Fig. 1). While this interface was inititally developed for remote parent-child communication, it was deemed suitable for the research as presented here as well. Nakama includes heat-pads, fits the cover story and attribution manipulation, and can be remotely controlled. A sender can remotely move the arms of the bear to gesture and touch. Each of the bear's shoulders contains two servo motors that enable movement of each arm in upward-downward and leftright direction. The sender can also wiggle the bear's ears and change the colors of its snout. Moreover, the bear can display the sender's heartbeats by means of a vibrotactile motor, and it can dissipate heat via a heat-pad embedded in its belly. For this study the heart rate was set to 70 BPM and the snout was green so that it, contrary to red or blue, would not have a direct association with warm or cold.

The heat-pad was either switched off (i.e., room temperature; on average $22.9^{\circ} \mathrm{C}$ ) or on. In the heated conditions, the temperatures as perceived on the participants' skin were in the range of $30-36^{\circ} \mathrm{C}$, which correspond with a mean temperature of $50.5^{\circ} \mathrm{C}$ as measured directly on the heatpad inside the bear [SD: 3.0, range $44.7-55.2^{\circ} \mathrm{C}$ (valid cases only)]. This range of perceived temperatures closely resembles actual human skin temperature which typically remains between 32 and $35^{\circ} \mathrm{C}$ [51]. Both the bear and room temperature were respectively logged with $10 \mathrm{~s}$ and $10 \mathrm{~min}$ intervals

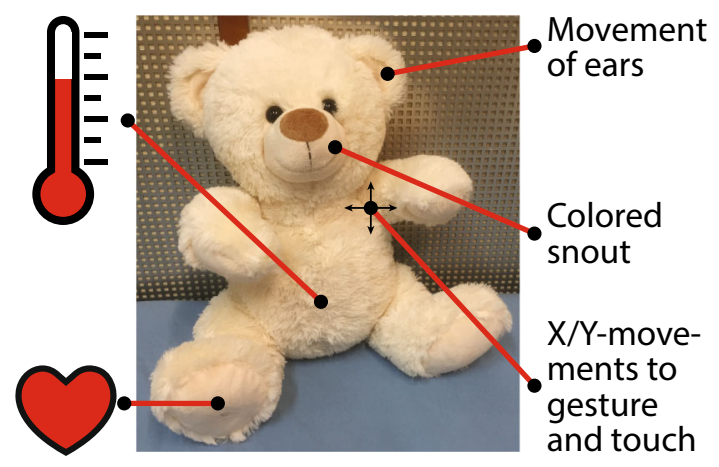

Fig. 1 The receiving side of Nakama with the several communication channels

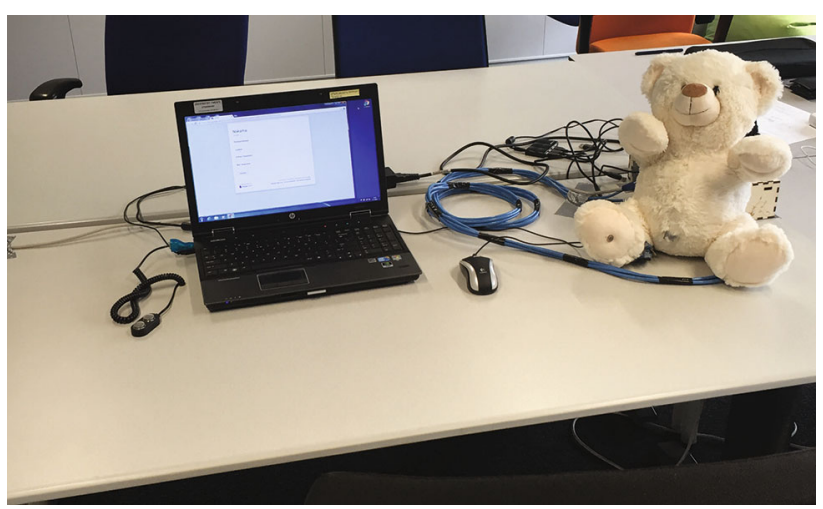

Fig. 2 The setup of study 1

by means of Maxim Integrated 'iButton' temperature loggers set to $0.0625^{\circ} \mathrm{C}$ accuracy. There are interpersonal differences in thermal sensitivity and the extent to which one considers a thermal stimulus 'warm' or 'cold', which are for example modulated by one's age and gender [52]. Moreover, thermal perception is subject to the ambient temperature and the physical qualities of the stimulus (e.g., size, body location, absolute temperature, or rate of change [51]). The thermal stimuli as presented in Study 1 (as well as in Study 2) were nonetheless deemed suitable as the temperature difference with the participant's skin was large enough to be well perceived and because both the ambient and bear temperatures were kept as constant as possible.

The participant was guided through the experiment by means of instructions and questionnaires in Google Forms. When the participant was instructed to hold the bear to receive the haptic messages, the experiment leader pressed a covered button to initiate a sequence of four stroking movements. These were rendered through up-and-down movements of the bear's right arm and applied to the participant's left arm. The experiment leader was present in the same room as the participant, seemingly minding his own business. We opted for this hide in plain sight solution to minimize the participant's suspicion regarding the communication partner being fictitious. The setting is depicted in Fig. 2.

\subsubsection{Measures}

We investigated three dimensions that have extensively been investigated in research on the physical-social warmth link and that were deemed relevant for affective CMC. These dimensions are the sense of social connectedness between the self and the interaction partner, perceptions of the personality of this other person, and perceived comfort. To investigate the social connectedness between the participant and the 'other', the Inclusion of Other in Self scale was utilized ('IOS'; [3]). Participants were asked which of seven to a different extent overlapping circle pairs best represented how close 
they felt to the other. The extent of overlap indicates the extent of social connectedness. This measure was successfully applied in research on physical warmth by IJzerman and Semin $[45,46]$. To investigate how physical warmth may affect perceptions of an interaction partner, participants were asked to assess the other person on ten Personality Traits (7-point bipolar scales, e.g., generous vs ungenerous), after reading an ambiguous description of this person. Five of the traits were related to the Warm-Cold dimension [4,31,102] and therefore expected to be affected by the manipulations, whereas the unrelated traits were expected to remain unaffected. This method is similar to the one applied by Williams and Bargh [102], who were among the firsts to propose the physical-social warmth link. The experienced comfort while receiving the haptic message was evaluated with the 'emotional attributes' of the Touch Perception Task ('TPT'; [35]). Participants were asked to indicate on a 5-point scale to what extent 14 terms such as irritating, soothing, or sensual described the experience they just had. The scores were aggregated to the factors Arousal and Comfort.

\subsubsection{Procedure}

The participant was welcomed and referred to the form on the laptop, in which Nakama and the other person were introduced: "Nakama has various communication channels such as moving arms and a colored snout that can be controlled by another participant. This other person can see you via the webcam. Moreover, this other person wears a sensor on the body, which measures heartbeat. You can feel this heartbeat in the bear." The attribution manipulation was added: "Nakama can display different temperatures, but for this study, the temperature is set at a constant level" (only applicable for the Room Temperature and Artificial Heat conditions). In the Body Heat condition the participant was led to believe that "besides the heartbeat, the sensor measures body temperature, which is also displayed via the bear". Next, the participant was asked to sign an informed consent form and to enter their age and gender. After viewing an instructional picture on how to hold the bear, the participant was asked to pick it up and hold it closely, after which the haptic message of approximately 30 s was initiated. We deliberately opted for a relatively brief message, as earlier research also demonstrated beneficial responses after a short exposure to warmth (e.g., $[28,102])$. Subsequently, the participant was asked to fill out the IOS, Personality Traits, and TPT questions, after which (s)he was thanked. A session took approximately 10 minutes.

\subsection{Results}

Data of four participants ( 3 male, 1 female) were omitted from the analyses as the recorded temperatures inside the bear deviated too much (> 2 standard deviations) from the average bear temperatures in the respective conditions. For the remaining 61 participants (Room Temperature: 20, Artificial Heat: 19, Body Heat: 22), the scores on all items were aggregated into the factors Personality Traits on the Warm/Cold dimension (Cronbach's $\alpha$ : .67) and Unrelated Traits $(\alpha:$.19) (following the factors as described by [102]), and the TPT factors: Arousal ( $\alpha: .78)$ and Comfort ( $\alpha: .86)$, as proposed by [35]. A one-way ANOVA on the IOS scores, with experimental group as independent variable did not yield significant effects: $F_{(2,58)}=0.76, p=.475$, partial $\eta^{2}=.025$. A one-way MANOVA including the scores on both types of personality traits, did not yield a significant difference between groups either: Wilks' $\Lambda=0.99, F_{(4,114)}=0.10, p=.983$, partial $\eta^{2}=.003$. No effects of the conditions on the Comfort and Arousal scores were found either, according to a one-way MANOVA: Wilks' $\Lambda=0.98, F_{(4,114)}=0.36$, $p=.834$, partial $\eta^{2}=.013$. Additionally, planned contrasts between the Room Temperature condition and the combined scores of both warm conditions were carried out, in order to investigate the effects of physical warmth as compared to room temperature. None of these contrasts yielded significant differences either (all $p s>$.670). The mean scores of the IOS, Personality Traits factors, and TPT factors are depicted in Fig. 3a-c.

\subsection{Discussion}

Although a growing body of research suggests that physical warmth can activate pro-social cognitions [43], and that this is a valuable implication for $\mathrm{CMC}$, our results do not corroborate this. No effects of warmth on social connectedness, personality perceptions, or comfort were found. Consequently, the attribution manipulation did not sort effects either. If physical warmth actually can activate pro-social cognitions in $\mathrm{CMC}$, this may only apply within specific boundary conditions. Here, we discuss potential boundary conditions that were not accounted for in Study 1, and we provide a rationale for Study 2, in which we investigate the effects of warmth embedded in a richer situational context.

A first possible boundary condition concerns the duration and absolute temperature of the thermal stimulus. This may have made the perception of the warmth more difficult and therefore less effective than in the earlier research that suggests the link between physical and social warmth. In said studies, participants were often exposed to the warmth for a longer period of time (e.g., [28,44,46,78,102]; ranging from approximately 1 to 15 minutes). The temperatures of the warm stimuli in these studies were likely higher than the body temperature simulations that participants perceived in our study. However, some studies also report effects after 


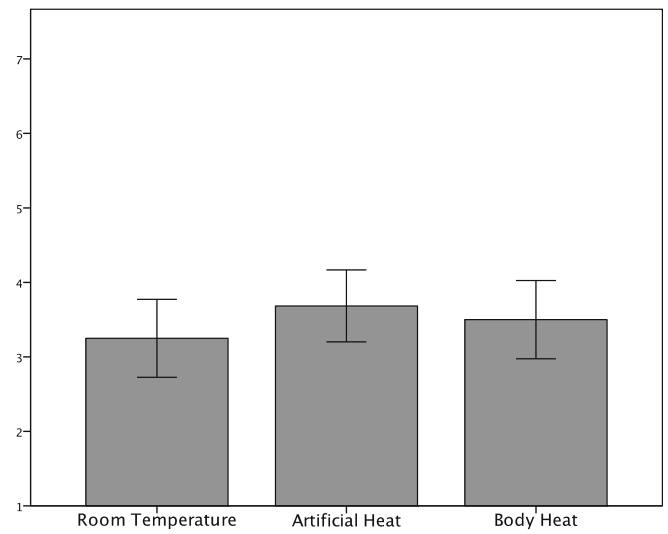

(a) IOS

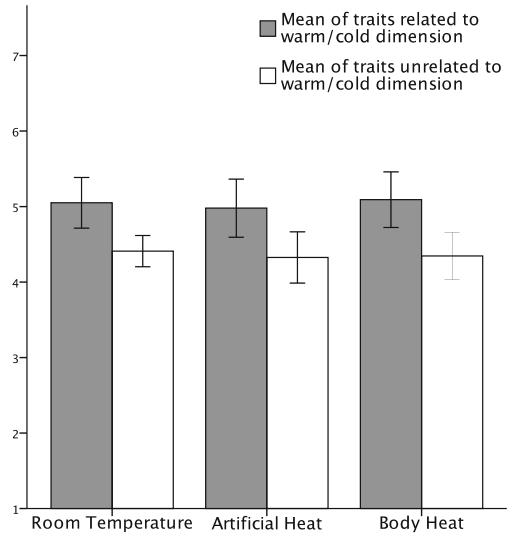

(b) Personality Traits

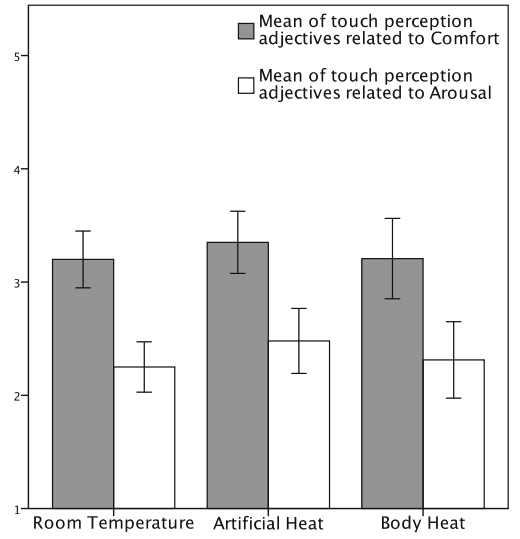

(c) Touch Perception

Fig. 3 Mean scores per condition for the IOS (a), Personality traits (b), and Touch perception task (c). Y-Axes represent the full range of possible scores and error bars indicate $95 \%$ confidence intervals

brief exposures to the heat (e.g., $[28,102]^{1}$ ), whereas nulleffects are reported despite longer durations [99]. In order to exclude this aspect as an explanation for the null-effects, participants in Study 2 will be exposed to the physical warmth for a longer period of time.

A second possible boundary condition which we did not consider in Study 1 concerns the context in which the message was sent, both in terms of the meaning of the message and the social context. Since participants did not know the fictitious interaction partner, they were unfamiliar with his/her intentions and the message could have been perceived without any apparent meaning. For a message to be interpreted, a shared understanding of its meaning is essential. This meaning also depends on the context and the complete conversation in which the message is embedded [36]. In line with this, and with real life touch interactions, the social context plays a significant role. Mediated touch communication may only be appropriate between actors in a close relationship $[33,73]$. Our second study will therefore take place in a contextually richer setting, where the intentions of the messages are clear. Similar to the paradigm of Cabibihan and Chauhan [13], participants will provide comforting touches to their partner during stressful circumstances.

Thirdly, personal characteristics such as one's need for social closeness ('attachment style' [7,11]), and/or for social touch ('touch receptivity' [10]) may have influenced the results. The physical-social warmth link supposedly originates from early intimate experiences during which also one's attachment style emerges, but these experiences and thus attachment styles can differ substantially per person. As a consequence, one's attachment style may have a modulating function in the effects of physical warmth. A higher need for attachment supposedly increases the sensitivity

\footnotetext{
${ }^{1}$ Note that these articles each report several studies, hence the seemingly contradicting claims.
}

to the physical-social warmth link $[28,44,95]$. We did not take this aspect into account in Study 1. The actual influence of attachment style is open for debate though. Some studies report effects of warmth without considering attachment style (e.g., [6,102]), whereas other studies failed to replicate the influence of attachment style [59,99]. We will nonetheless take attachment style, as well as touch receptivity, into account as a potential boundary condition for Study 2.

\section{Study 2: Communicating with one's partner}

In the second study, romantic couples were invited to evaluate a new communication system, namely Nakama. The couples were led to believe they would watch a scary movie simultaneously, albeit in separate rooms. During viewing, one person of the dyad could send comforting messages to the receiving partner via Nakama. This paradigm is partly based on the premise that real human touches can alleviate stress responses [17,23], and on earlier mediated touch research by Cabibihan and Chauhan [13]. The roles of sender and receiver were switched for a second movie. In reality, the sender and receiver watched the two movies crosswise, and all messages through Nakama were scripted, in order to make sure every participant was exposed to the same stimuli. Unknown to the participants, the buttons that were pressed on the controller by the sender thus did not have any influence on the messages. This setup allowed us to investigate the effects of warmth and attribution over a longer period and in a relatively rich situational context. That is, the haptic messages were sent in the social context of a romantic relationship with the intention to provide comfort during stressful circumstances; a potential application of affective haptics. Moreover, additional subjec- 


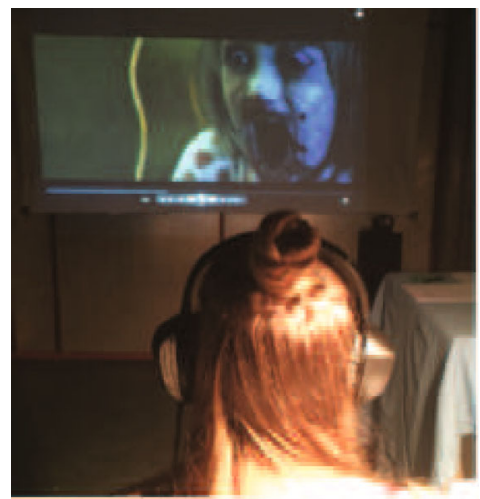

(a) Movie

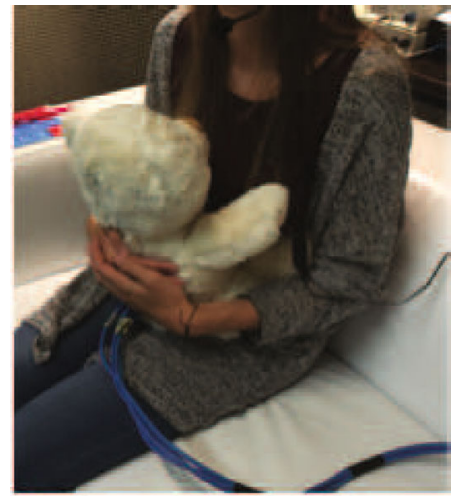

(b) Bear

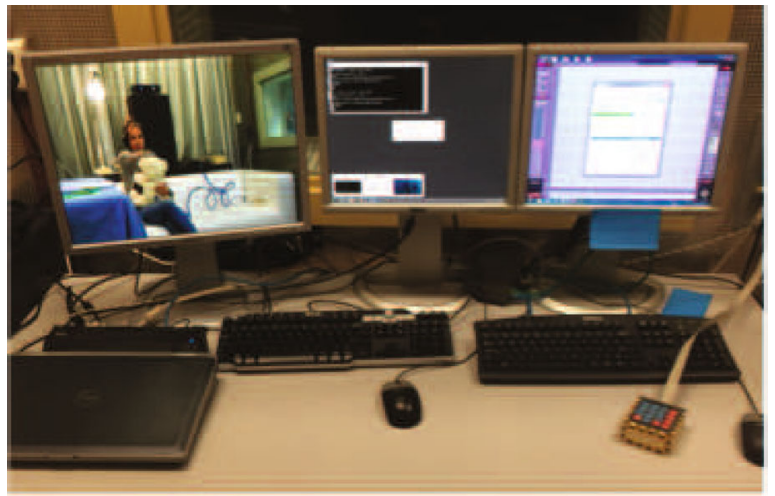

(c) Control Room

Fig. 4 The experimental setting with the movie (a), a participant holding Nakama (b), and the control room (c)

tive and physiological measures were included to gain more insights in the opportunities and limitations of the physicalsocial warmth link for CMC, and to verify the influence of personality characteristics such as attachment style and touch receptivity.

\subsection{Methods}

\subsubsection{Participants}

In total, 31 heterosexual romantic couples participated in the experiment. The mean age was 51.9 (SD: 17.0, range 1974). Couples were randomly assigned to either the Room Temperature (10), Artificial Heat (11), or Body Heat (10) condition. Every participant indicated to have (corrected to) normal vision and hearing, and received $€ 30$ - plus travel expenses for participation. This study was reviewed by the internal review board of [institution] and carried out in accordance with the Helsinki Declaration of 1975, as revised in 2013 [105]. Participants gave written informed consent prior to the experiment.

\subsubsection{Setting and apparatus}

Two adjacent lab rooms of ca. $2.5 \times 3 \mathrm{~m}$, with an average temperature of $20.0^{\circ} \mathrm{C}$, were furnished and decorated similarly. When the participants assumed the receiver role, and thus were holding Nakama, they watched the movie "The Descendent" [2], whereas "Red Balloon" [92] was displayed when participants assumed the sender role. The movies were projected with a $1024 \times 768$ px resolution on a $1.5 \times 1 \mathrm{~m}$ sized screen on the wall. As suggested in [71], excerpts from a neutral aquatic movie, "Coral Sea Dreaming: Awaken" [37] were displayed prior to both movies, in order to bring people quickly (back) to their physiological baseline level. The participants sat on a couch, approximately $2 \mathrm{~m}$ from the screen, while wearing headphones for the movies' audio and to mask the sounds of Nakama's motors. The experiment leader overviewed both rooms from an adjacent control room via cameras and a monitor (see Fig. 4 for an impression of the setting).

The experiment leader initiated eight messages via the bear. Two messages consisted of ear wiggling, one of a color change from green to yellow, and five events comprised touches. Three of the touch sequences consisted of three up-and-down stroking movements each, whereas the other two sequences contained nine up-and-down strokes each. As the cover story comprised the evaluation of Nakama as communication medium, including a small amount of 'messages' was deemed essential. Excluding all messages could imply that the partner did not have any intentions to comfort the receiver, which in turn would bias the results. The average temperature of the bear in the warm conditions was $48.0^{\circ} \mathrm{C}\left(S D: 5.4\right.$, range $37.3-57.7^{\circ} \mathrm{C}$, valid cases only). Physiological stress responses were recorded with the BioSemi ActiveTwo system (http://www.biosemi.com), with passive Nihon Kohden electrodes for the Galvanic Skin Response ('GSR') and active flat Ag-AgCl electrodes to measure cardiac activity. Physiological data were logged by means of Actiview software (v7.06), with a sampling rate of 2048 Hz. Markers to synchronize the physiological responses with movie and messages sent through Nakama were placed automatically, via custom-built software.

\subsubsection{Measures}

Since physical warmth may elicit responses on different dimensions, we applied a series of measures and categorized them in Social Warmth, Communication Experience, and Emotional and Physiological Comfort. Personal Characteristics were recorded as well. Contrary to Study 1, all questionnaires of Study 2 were in Dutch. 
Social warmth To investigate how the temperature and attribution would affect the perception of the partner as well as the perceived social connectedness, the same measures as in Study 1 (i.e., IOS [3] and Personality Traits [102]) were applied. Since the questions in Study 2 were formulated to assess the partner, the general ambiguous description as applied in Study 1 (see: Sect. 2.1.3) was omitted. An additional IOS scale to assess the perceived intimacy, and the Presence-in-Absence ('PiA') Subscale of the Affective Costs and Benefits in Communication Technology questionnaire ('ABCCT'; [107]) were included as well. The PiA consists of three statements such as "Communicating with my partner using Nakama helps me feel closer to my partner", to be answered on a 5-point likert scale.

Communication experience We investigated to what extent participants felt that communication via Nakama could provide Social Support ('SS'; 5 items) and allowed Emotional Expression ('EE'; 3 items). These subscales are particularly applicable, as the ABCCT [107] was specifically developed to evaluate communication media on different dimensions, after usage in a daily life context. Moreover, the perceived Arousal and Comfort were measured again (TPT; [35]).

Emotional and physiological comfort Besides the affective experience related to communication, we also wanted to gain insights in the participant's emotional state. To this end, we applied both subjective and objective physiological measures. To record the perceived emotional state both prior to and after the movie in which participants received messages, the Self Assessment Manikin ('SAM'; [12]) and a validated Dutch translation [70] of the Positive and Negative Affect Schedule ('PANAS'; [97]) were utilized. The SAM is a 9-point pictorial scale to measure Valence, Arousal, and Dominance and the PANAS indicates one's levels of Positive and Negative Affect by means of 5-point ratings of 20 adjectives related to affective state. Moreover, participants were asked how scary, respectively thrilling they experienced the movie (11-point scale: ' 0 ' = not at all, ' 10 ' = very much) $[21,74]$. Additionally, we recorded the participant's GSR, which is linearly correlated with arousal [57] and located the electrodes at the palm and on top of the first lumbrical muscle of the left hand. Moreover, Heart Rate ('HR') and Heart Rate Variability ('HRV')-i.e., the temporal differences between successive inter-beat intervals in the electrocardiogram ('ECG') [85] — were measured by means of two electrodes that were placed on the right clavicle and the left floating rib. When one is more aroused, the HR increases [64] and HRV decreases. We utilized the Root Mean Square of Successive Differences ('RMSSD') as measure for HRV. The proposed range of objective and subjective measures was considered suitable to provide a coherent and reliable image of one's emotional state, and thus on how warmth and the attribution may affect this state. The mean GSR, HR, and HRV were determined for a 2 minute baseline period while watching the neutral movie, and for the duration (10:47) of the scary movie excluding the non-scary introductory scenes.

Personal characteristics Besides the age and gender, the Attachment Style [7] of the participant was assessed. Each participant indicated on a 7-point likert scale how descriptive each of four short Dutch paragraphs $[26,65]$ was for him or her. The most descriptive paragraph represented the participant's attachment style (either secure, dismissing, preoccupied, or fearful). In line with IJzerman et al. [44], who amongst others suggest the modulating effect of attachment style on the physical-social warmth link, we recoded the latter three as 'insecure'. Participants also reported their Touch Receptivity with 10 7-point items ('TR'; [10]). The Dutch Marital Satisfaction and Communication Questionnaire [94] was included to measure relationship strength, as this may modulate the responses to touch [17]. Participants were asked how applicable (' 1 ' = 'Not', '7' = 'Highly') 16 statements regarding the subscales Marital satisfaction, Negative communication, and Open communication were.

\subsubsection{Procedure}

Each couple was introduced to a demonstration version of Nakama, and to the applicable temperature attribution in a similar way as in Study 1. To emphasize the cover story that Nakama is a real-time display of heartbeats, the experiment leader wore a physiology sensor on the wrist and explained that his cardiac activity was displayed in real time. In the Body Heat condition, participants were led to believe that the experiment leader's body heat was sensed and displayed similarly. Participants were encouraged to feel the signals in the bear and they were told that the electrodes to measure their physiological responses would also convey the heartbeats, and in the Body Heat condition also the warmth (to this end, a bogus temperature sensor was attached in the Body Heat condition). After a briefing on the procedure and the aim of the study (i.e., "investigate how Nakama can be applied and how it is experienced"), participants signed an informed consent form and moved to separate rooms where the crosswise procedure started. The sequence of activities, questionnaires, instructions, and movie watching is listed in Table 1, as are the respective roles (i.e., sender or receiver) each participant assumed during each session. The receiving participant held the bear directly to his/her body throughout the movie, in order to ensure a long exposure to the warmth. The sending participant was instructed to convey messages at his/her own discretion throughout the movie. The 'sender-movie' was respectively followed by a few bogus questions (not reported) and by a neutral movie to bring the sender's arousal level back to a baseline level. The session took approximately $1 \mathrm{~h} 40$, and 
Table 1 The procedure of study 2 with the various questionnaires (Q) and the accompanying measures

\begin{tabular}{|c|c|c|}
\hline \multirow{4}{*}{$\begin{array}{l}\text { Preparation } \\
\text { (ca. } 25 \mathrm{~min})\end{array}$} & \multicolumn{2}{|c|}{$\begin{array}{c}\text { Room 1: } \\
\text { Welcome; introduction; Nakama demonstration; verbally emphasizing manipulations; informed consent }\end{array}$} \\
\hline & $\begin{array}{l}\text { Room 1: Participant } 1 \text { as sender (controller) } \\
\text { Attach electrodes }\end{array}$ & $\begin{array}{l}\text { Room 2: Participant } 2 \text { as receiver (with bear) } \\
\text { Q1: Demographics, Touch Receptivity, Attachment } \\
\text { Style, Relationship Satisfaction, SAM, PANAS }\end{array}$ \\
\hline & $\begin{array}{l}\text { Q1: Demographics, Touch Receptivity, Attachment } \\
\text { Style, Relationship Satisfaction }\end{array}$ & Attach electrodes; instructions on bear holding \\
\hline & Instructions on controller use & Wait \\
\hline \multirow{3}{*}{$\begin{array}{l}\text { Session } 1 \\
(\text { ca. } 30 \mathrm{~min})\end{array}$} & Start movie sequence: 3 min. baseline video & $\begin{array}{l}\text { Start movie sequence: } 3 \mathrm{~min} \text {. baseline video }+ \text { phys- } \\
\text { iological recordings }\end{array}$ \\
\hline & $\begin{array}{l}\text { Scary movie "Red Balloon"; (supposedly) sending } \\
\text { messages by means of controller }\end{array}$ & $\begin{array}{l}\text { Scary movie "The Descendent"; receiving eight mes- } \\
\text { sages; experimental physiological recordings }\end{array}$ \\
\hline & $\begin{array}{l}\text { Q2: Bogus sender questions; neutral video to return } \\
\text { to baseline }\end{array}$ & $\begin{array}{l}\text { Q2: IOS's, Personality Traits, TPT, SAM, PANAS, } \\
\text { Movie perception }\end{array}$ \\
\hline \multirow{2}{*}{$\begin{array}{l}\text { Preparation } \\
\text { Session } 2 \\
\text { (ca. } 5 \mathrm{~min})\end{array}$} & \multicolumn{2}{|c|}{ Experiment leader swaps bear for controller and vice versa, while participants stay in their rooms } \\
\hline & $\begin{array}{l}\text { Room 1: Pp1 as new receiver (with bear) } \\
\text { Q3: SAM, PANAS; instructions on bear holding }\end{array}$ & $\begin{array}{l}\text { Room 2: Pp2 as new sender (controller) } \\
\text { Instructions on controller use }\end{array}$ \\
\hline \multirow[t]{3}{*}{$\begin{array}{l}\text { Session } 2 \\
(\text { ca. } 35 \mathrm{~min})\end{array}$} & $\begin{array}{l}\text { Start movie sequence; } 3 \text { min. baseline video + phys- } \\
\text { iological recordings } \\
\text { Scary movie "The Descendent"; receiving eight mes- } \\
\text { sages; experimental physiological recordings }\end{array}$ & $\begin{array}{l}\text { Start movie sequence; } 3 \text { min. baseline video } \\
\text { Scary movie "Red Balloon"; (supposedly) sending } \\
\text { messages by means of controller }\end{array}$ \\
\hline & $\begin{array}{l}\text { Q4: IOSs, Personality Traits, TPT, SAM, PANAS, } \\
\text { Movie perception, ABCCT }\end{array}$ & Q3: Bogus sender questions, ABCCT \\
\hline & Detach electrodes & Detach electrodes \\
\hline $\begin{array}{c}\text { End } \\
\text { (ca. } 5 \mathrm{~min})\end{array}$ & $\begin{array}{r}\text { Control } \\
\text { Joint debriefing; administra }\end{array}$ & $\begin{array}{l}\text { Room: } \\
\text { tional formalities; thanking }\end{array}$ \\
\hline
\end{tabular}

was closed with a debriefing on the deceptive elements in the study.

\subsection{Results}

\subsubsection{Preprocessing}

Two female participants indicated to have difficulties with comprehending the questionnaires, and the temperature manipulation failed for one male participant. Their data, but not the data from their partners, were considered invalid and therefore omitted from all analyses (remaining $n$ per group: Room temperature: 19, artificial heat: 22 , body heat: 18 ). Due to technical problems with the physiological recordings, emotional state data from six additional participants were omitted from that analysis $(n=18,18$, and 17, respectively).

The physiological measurements were processed with MATLAB R2013b (http://www.mathworks.com) and imported with the FieldTrip toolbox [68]. By means of a Fast Fourier Transform, the low frequency components in the ECG were filtered out, after which the HR and HRV were derived with a peak-detection algorithm. Moreover, as suggested by Lykken and Venables [62], range correction was applied on the GSR data. Each GSR data point was divided by the maximum GSR value for each individual and the mean GSR was computed subsequently. The scores of all subjective items were aggregated into the appropriate factors.

\subsubsection{Stress elicatation}

A repeated measures with recording moment as independent, within-subjects variable (two levels: baseline and experiment) MANOVA was carried out on the objective (HR, HRV, and GSR), and on the subjective (PANAS and SAM subscales) data of the 53 valid cases, to verify whether the movie indeed increased arousal as compared to the baseline levels. The internal consistencies of both the pre- and post-movie Positive Affect (Cronbach's $\alpha$ : .86 and .84 , respectively) and Negative Affect ( $\alpha: .82$ and .85, respectively) scores were good. The HRV data were $\log _{10}$-transformed to correct for the violation of the normality assumption. The analysis yielded a significant main effect of recording moment: Wilks' $\Lambda=.47, F_{(8,45)}=6.36, p<.001$, partial $\eta^{2}=.531$. Subsequent investigations of the repeated measures ANOVAs of each dependent variable (see Table 2) indicated that the GSR, subjective Arousal (SAM), and Negative Affect significantly increased due to the scary movie, whereas the Valence and Positive Affect significantly decreased, as compared to the baseline. 
Table 2 Stress elicitation results

\begin{tabular}{lllllllll}
\hline & HR $(\mathrm{BPM})$ & HRV (RMSSD) & GSR & Valence (SAM) & Arousal (SAM) & Dominance (SAM) & Positive affect & Negative affect \\
\hline Baseline & 68.04 & 1.48 & $\mathbf{. 2 1}$ & $\mathbf{6 . 9 4}$ & $\mathbf{3 . 2 5}$ & 5.53 & $\mathbf{3 0 . 8 9}$ & $\mathbf{1 2 . 6 0}$ \\
Experiment & 67.29 & 1.53 & $\mathbf{. 3 3}$ & $\mathbf{5 . 4 0}$ & $\mathbf{4 . 6 4}$ & 5.06 & $\mathbf{2 8 . 5 1}$ & $\mathbf{1 5 . 6 2}$ \\
$p$-value & .117 & .166 & $<\mathbf{. 0 0 1}$ & $<. \mathbf{0 0 1}$ & $<.001$ & .108 & $\mathbf{0 1 5}$ & $<\mathbf{. 0 0 1}$ \\
\hline
\end{tabular}

Bold-faced values indicate significant effects

Table 3 Means and SDs (in brackets) per condition for the emotional and physiological state measures

\begin{tabular}{lllllllllll}
\hline & HR (BPM) & HRV (RMSSD) & GSR & Valence & Arousal & Dominance & Positive affect & Negative affect & Scary & Thrilling \\
\hline Room temp. & -1.36 & -1.28 & 0.12 & -2.00 & 1.61 & -0.50 & -2.50 & 4.17 & 5.75 & 5.83 \\
& $(3.21)$ & $(21.00)$ & $(0.22)$ & $(2.40)$ & $(2.00)$ & $(1.95)$ & $(5.24)$ & $(5.93)$ & $(2.35)$ & $(2.46)$ \\
Artificial heat & -0.39 & 3.46 & 0.06 & -1.28 & 1.00 & -0.39 & -2.33 & 2.22 & 4.47 & 5.11 \\
& $(4.65)$ & $(29.95)$ & $(0.24)$ & $(2.35)$ & $(2.38)$ & $(2.45)$ & $(9.27)$ & $(5.06)$ & $(2.83)$ & $(2.70)$ \\
Body heat & -0.49 & 7.32 & 0.17 & -1.35 & 1.58 & -0.53 & -2.00 & 2.65 & 5.24 & 5.47 \\
& $(1.89)$ & $(14.27)$ & $(0.20)$ & $(1.66)$ & $(2.15)$ & $(1.97)$ & $(5.16)$ & $(4.31)$ & $(2.49)$ & $(2.32)$ \\
\hline
\end{tabular}

$\Delta$-Values represent the difference between the experimental and baseline measures

\subsubsection{Emotional and physiological comfort}

To investigate how the warmth and attribution manipulations affected the emotional state of the same 53 participants, we computed the differences between the untransformed experimental measures and their baseline counterparts for all aforementioned dependent variables. Subsequently, a oneway MANOVA was carried out on these difference scores and on the Scariness and Thrillingness scores, with the experimental group (i.e., Room Temperature, Artificial Heat, and Body Heat) as independent between-subjects variable. The analysis did not demonstrate a significant difference between any of the groups: Wilks' $\Lambda=.79, F_{(20,82)}=0.51$, $p=.953$, partial $\eta^{2}=.111$. Additional planned contrasts between the Room Temperature and the two warm conditions combined did not yield significant differences either. All Emotional State scores are listed in Table 3.

\subsubsection{Social warmth}

The two IOS scores, the Personality Traits related to the Warm-Cold dimension (Cronbach's $\alpha: .75$ ) as well as the unrelated traits $(\alpha: .59)$, and the Presence-in-Absence subscale $(\alpha: .75)$ were included as dependent variables in a MANOVA, with experimental group (i.e., Room Temperature, Artificial Heat, and Body Heat) as independent variable. No effects of the experimental group on the perceptions of the partner could be found. Wilks' $\Lambda=.93, F_{(10,104)}=0.38$, $p=.951$, partial $\eta^{2}=.036$. This absence of significant differences also applied in the planned comparisons between the warm conditions and the Room Temperature condition.

\subsubsection{Communication experience}

The aggregated Arousal, Comfort, Emotional Expression, and Social Support scores (Cronbach's $\alpha$ s, respectively: .85, $.90, .65$, and .85) were included in a MANOVA with the three groups as independent variable. A significant effect of the experimental condition was found: Wilks' $\Lambda=.74$, $F_{(8,106)}=2.11, p=.041$, partial $\eta^{2}=.137$. Inspection of the individual ANOVAs revealed a significant effect on Emotional Expression: $F_{(2,56)}=5.38, p=.007$, partial $\eta^{2}=.161$. Pairwise comparisons demonstrated that Body Heat was considered to provide more opportunities for emotional expression than Room Temperature $(p=.002)$ and Artificial Heat $(p=.049)$. The means of the Social Warmth and Communication Experience measures can be found in Table 4.

\subsubsection{Interpersonal differences}

When included as covariates in MANCOVAs, neither Touch Receptivity (Cronbach's $\alpha:$.69), Marital satisfaction ( $\alpha: .84)$, Negative communication $(\alpha: .84)$, nor Open communication $(\alpha: .71)$ did affect the interpretation of the effects of physical warmth and the attribution on the various measures. These covariates are therefore not further reported. Two-way MANOVAs were carried out with attachment style (secure, insecure) and the experimental group as independent variables, and the same sets of dependent variables as reported before. The division of secure and insecure participants for the Room Temperature, Artificial Heat, and Body Heat groups was $8 / 10,11 / 7$, and 10/7, respectively, for the Emotional State analyses, and 9/10, 12/10, and 11/7, respectively, for the remaining analyses. Neither main effects 
Table 4 Means and SDs (in brackets) per condition for the social warmth and communication experience measures

\begin{tabular}{|c|c|c|c|c|c|c|c|c|c|}
\hline & \multicolumn{2}{|l|}{ IOS } & \multicolumn{2}{|c|}{ Personality traits } & \multicolumn{3}{|c|}{ ABCCT } & \multicolumn{2}{|l|}{ TPT } \\
\hline & Closeness & Intimacy & Temp. & Unrelated & $\mathrm{PiA}$ & $\mathrm{EE}$ & SS & Arousal & Comfort \\
\hline Room temperature & $\begin{array}{l}4.68 \\
(1.38)\end{array}$ & $\begin{array}{l}4.63 \\
(1.46)\end{array}$ & $\begin{array}{l}4.60 \\
(1.00)\end{array}$ & $\begin{array}{l}4.68 \\
(0.88)\end{array}$ & $\begin{array}{l}3.39 \\
(1.12)\end{array}$ & $\begin{array}{l}2.93 \\
(0.91)\end{array}$ & $\begin{array}{l}3.03 \\
(1.05)\end{array}$ & $\begin{array}{l}2.26 \\
(0.74)\end{array}$ & $\begin{array}{l}3.26 \\
(0.74)\end{array}$ \\
\hline Artificial heat & $\begin{array}{l}4.59 \\
(1.79)\end{array}$ & $\begin{array}{l}4.64 \\
(1.79)\end{array}$ & $\begin{array}{l}4.83 \\
(1.14)\end{array}$ & $\begin{array}{l}4.66 \\
(0.86)\end{array}$ & $\begin{array}{l}3.68 \\
(0.92)\end{array}$ & $\begin{array}{l}3.32 \\
(1.01)\end{array}$ & $\begin{array}{l}3.14 \\
(1.21)\end{array}$ & $\begin{array}{l}2.55 \\
(0.81)\end{array}$ & $\begin{array}{l}3.48 \\
(0.87)\end{array}$ \\
\hline Body heat & $\begin{array}{l}4.78 \\
(1.59)\end{array}$ & $\begin{array}{l}5.06 \\
(1.39)\end{array}$ & $\begin{array}{l}4.89 \\
(0.92)\end{array}$ & $\begin{array}{l}4.69 \\
(0.77)\end{array}$ & $\begin{array}{l}3.63 \\
(0.91)\end{array}$ & $\begin{array}{l}3.89 \\
(0.70)\end{array}$ & $\begin{array}{l}3.47 \\
(0.83)\end{array}$ & $\begin{array}{l}2.53 \\
(0.76)\end{array}$ & $\begin{array}{l}3.59 \\
(0.85)\end{array}$ \\
\hline
\end{tabular}

Bold-faced values indicate significant effects

Table 5 Results of meta-analyses of the warmth and attribution effects for all recurring measures

\begin{tabular}{|c|c|c|c|c|c|c|c|c|}
\hline & \multicolumn{4}{|c|}{ Warmth manipulation } & \multicolumn{4}{|c|}{ Attribution manipulation } \\
\hline & $d$ & $95 \% \mathrm{CI}$ & $Z$ & $p$ & $d$ & $95 \% \mathrm{CI}$ & $Z$ & $p$ \\
\hline IOS & 0.16 & {$[-0.22,0.54]$} & 0.82 & .415 & 0.14 & {$[-0.30,0.57]$} & 0.61 & .541 \\
\hline Personality traits (warm/cold) & 0.13 & {$[-0.25,0.51]$} & 0.66 & .511 & 0.10 & {$[-0.34,0.54]$} & 0.44 & .659 \\
\hline Arousal (TPT) & 0.29 & {$[-0.09,0.68]$} & 1.50 & .135 & 0.13 & {$[-0.31,0.57]$} & 0.59 & .553 \\
\hline Comfort (TPT) & 0.22 & {$[-0.16,0.60]$} & 1.12 & .261 & 0.17 & {$[-0.27,0.60]$} & 0.74 & .462 \\
\hline
\end{tabular}

of attachment style (all $p \mathrm{~s}>.167$ ), nor interaction effects between attachment style and experimental condition (all $p \mathrm{~s}$ $>$.187) were found.

\section{Meta analyses and post-hoc power tests}

Although none of the between-subjects effects reached statistical significance, the majority of the scores did emerge in the hypothesized direction. As one of the criticisms regarding the supposed effects of warmth concerns the power of the studies [43], we decided to conduct random-effect model meta-analyses on the recurring measures of Study 1 and 2 (i.e., IOS, Warm/Cold Personality Traits, and Arousal and Comfort (TPT)), in order to increase the power. The effects of warmth (i.e., Room Temperature vs warm conditions combined; $n=120$ ) and attribution (i.e., Artificial Heat vs Body Heat; $n=81$ ) were investigated. The results of the metaanalyses corroborate the findings of the individual studies, as no significant effects were found for any of the measures (see Table 5).

Moreover, post-hoc power analyses were carried out to provide a better understanding of the practical significance of the effects. The effect sizes of all statistical tests of Study 1 and 2, computed with G*Power [27], can be considered small at best, according to the conventions by Cohen [18]. Given the small effect sizes, the power $(1-\beta)$ to actually detect effects varied between 0.08 and 0.34 , with the exception of the Communication Experience test in Study 2, of which the effect size was medium and the power 0.88 . With the power set at the recommended 0.80 [18], the required sample sizes for the tests would have to increase to at least $n=123$, and even to $n=1188$ for the least powered test, for the group differences to reach statistical significance. This means that even if the effect would reach significance for large samples, its practical relevance is still limited.

\section{General discussion}

With two studies and meta-analyses, we set out to find empirical support for the suggestion that interaction via a physically warm medium may increase comfort and pro-social cognitions about one's communication partner, as compared to interaction via an unheated medium [9,33,47,72,87]. We intended to verify whether the physical-social warmth link is also applicable in CMC. To further inform the design of haptic communication devices, we also discerned between warmth that is attributed to non-social, inanimate technology, and warmth that has inherent social connotations [i.e., the body heat of a stranger (Study 1) or one's own partner (Study 2)]. Although the participants thought that a device that mediates body temperature provided more opportunities for emotional expression, this was not reflected in other effects of physical warmth. Despite the longer exposure to the warmth, a richer situational context, and the consideration of individual personality characteristics in Study 2, neither the temperature nor the attribution manipulation did alter perceptions of closeness to the interaction partner or pro- 
vided additional emotional or physiological comfort during stressful circumstances. Our results are in line with earlier failed attempts to find support for the existence of the physical-social warmth link $[24,25,63,99]$ and the supposed modulating role of attachment style [59,99], but contradict a growing amount of studies that suggest the existence of this link [43]. Here, we discuss our methodology and findings and relate these to earlier research on the physical-social warmth link. We will argue that contrary to what is often suggested, this link may not necessarily be suitable for affective CMC.

The absence of the hypothesized effects of physical warmth may be explained by the medium that was used. First of all, Nakama may have been perceived as childish which may have reduced the overall impressions of the messages. Moreover, due to its design, the effects of warmth on comfort and social connectedness could not be investigated in isolation. That is, holding a soft object during communication may in itself already alleviate stress responses. A decrease in cortisol level (i.e., the "stress-hormone") was for instance found while hugging a pillow during a phone call $[81,106]$, and interactions with soft robotic plush animals increased the "love-hormone" oxytocin [84] and had beneficial effects on coping with stress on the longer term as well [96]. There is no added value of warmth, over simply holding a bear at room temperature, with regard to stress reduction. It is however unlikely that the childishness and potential physical comfort provided by Nakama also account for the absence of physical-social warmth effects. One may of course suggest that (a combination of) the mediated touches, heartbeats, and perhaps the other channels have occluded the effects of physical warmth on social connectedness, as these signals may have increased the social connectedness by themselves $[26,36,40,50]$. This suggested ceiling effect seems however unlikely considering the 'mildly warm' perceptions of the interaction partner (according to the scores in both studies); the influence of the other communication channels seems negligible. We can conclude that if warmth has positive effects on comfort and/or social warmth, it does not prevail over the other physical qualities and communication channels of Nakama. An additional caveat that has to be placed is the fact that sensitivity to thermal changes differs from person to person (e.g., [51,52], see also Sect. 2.1.2). While it is unlikely that these interpersonal differences affected the outcomes of our studies (as earlier research on the physical-social warmth link did also present generic thermal stimuli), future research could benefit from a paradigm in which thermal changes are applied in reference to the participant's current body temperature (e.g., [76]).

After Study 1, stricter boundary conditions within which physical warmth in CMC supposedly could induce effects were proposed. In line with earlier research (e.g., [28,99, 102]) the results of our studies suggest that the duration of the exposure to the warmth is not an essential boundary con- dition. It may however be the case that the contrast between the warmth and room temperature was not large enough to detect effects. That is, the majority of studies in favor of the physical-social warmth link discern between warm and cold conditions (e.g., [28,44,102]), and coldness supposedly has a negative impact on pro-social cognitions, rather than warmth having a positive impact $[6,53,99]$. People may have a default orientation towards others that can be described as 'mildly warm' [6], and likely a warmer attitude towards their own partners; a claim that can also be supported by the scores in both studies. Additional physical warmth may not change this default as much as coldness can [6]; in particular because avoidance motivations towards the social world (which in this case would be represented by coldness) are usually stronger than approach motivations (or warmth) $[6,14]$. This would not necessarily mean that warmth has no effects, but that the added value of it can mainly be found in its restorative or compensatory effects in cases of social stress, such as feelings of loneliness, guilt, or exclusion, or with psychological pathologies $[6,39,43,55,72,93]$. Despite the introduction of a clear context for the haptic messages in Study 2, namely comforting the partner in a situation of stress, no social stress was induced. Moreover, the participants knew that their partner was literally close by and directly available after the scary movie. Since we did not actively induce social stress, or at least control for it, one may say that our methodology was not suited to actually detect the supposed effects of warmth in CMC. This argument does not seem sound however, as the majority of studies that report positive effects of physical warmth do not include a social stress context either (e.g., $[28,33,45,78,102])$. If the physical-social warmth link actually would be applicable in CMC, we would at least have found some indications that warmth positively influenced social cognitions.

Although there is a growing body of evidence in support of the physical-social warmth link, neither the underlying mechanisms nor the boundary conditions are fully understood yet; the research is still in its infancy [42,43]. Moreover, some of the studies may have had a participant sample that was too small to actually support the tested hypotheses, which could have resulted in possible publication bias or even false positives [43]. Although the sample sizes in our studies and meta-analyses ( $n=65,62,120$, and 81 , respectively) can be considered substantial, they appeared, according to our posthoc power analyses, too small to actually demonstrate effects of warmth and the attribution thereof. This may simply be because the effects, if these actually exist, do not seem to be as outspoken as suggested. Moreover, as mentioned, there have been several attempts, often with significantly larger sample sizes, to exactly replicate results (e.g., [24,25,59,63] or to find similar effects (e.g., [99]) that were unsuccessful. Abovementioned considerations raise the question whether the supposed effect actually exists, and if so, to what extent 
these significant findings are of actual practical use. Our results suggest, contrary to earlier research $[9,33,47,72,87]$, that the effects on comfort and social warmth, if any, are not substantial enough to be of practical significance during CMC. This is however not to say that we should disregard warmth as a communication medium altogether. Valuable insights in the conveyance of emotions through different thermal patterns have been gained in earlier research (e.g., $[60,61,76])$. However, the elicitation of beneficial responses in CMC through the use of warmth should be based on other principles (e.g., abstract thermal messages) than on the physical-social warmth link.

Although the majority of the results suggest that physical warmth does not induce outspoken social responses in CMC, participants did indicate that mediated body heat increased the perceived opportunity for emotional expression. These findings may seem contradicting. It could however be the case that the participants considered the option to switch the non-social, inanimate temperature on or off merely as an attribute of the device, whereas the conveyance of body heat may have been considered an actual social communication channel. Having this additional communication channel could have increased the perceived 'richness' of the device i.e., the extent to which a medium facilitates the conveyance of immediate feedback and contextual information [20]and may therefore have provided more options for emotional expression. Even though it is not reflected in the actual social cognitions, this finding suggests that in order to advance the understanding of the design of affective haptic communication devices, it is indeed valuable to discern between responses that are elicited by the physical qualities of haptic interfaces and those informed by the social meaning of the signals.

\section{Conclusions}

Based on the results from our studies and meta-analyses, which could not confirm any of the hypotheses, as well as on the doubts and criticism regarding the suggested physicalsocial warmth link, we conclude that physical warmth does not have clear beneficial effects with regard to feelings of social connectedness, social warmth, or comfort in a setting of CMC. The effects of the physical-social warmth link, if any, seem too small to be of actual practical use. Warmth does, according to the physical-social warmth link, not induce significantly stronger effects than alternative affective haptic communication channels such as mediated touch or heartbeats. If the supposed beneficial effects of the physicalsocial warmth link actually exist, they may only appear in CMC under very specific circumstances that go beyond the ones that were considered in our studies. In order to advance the development of effective affective haptic interfaces, it is not only important to understand the opportunities that these interfaces offer, but also their limitations. From the latter perspective, the work as presented here thus provides valuable knowledge despite the unsupported hypotheses. Another valuable insight provided by this work is the suggestion that research on affective haptic interfaces in general, not only interfaces that include warmth, should discern between the inherent hedonic qualities of haptic actuators, and stimulation with haptics that have social meaning such as social touch technology. A thorough understanding of the capabilities of different (combinations of) haptic actuators and attributions, and of how these stimuli are cognitively processed, may inform the design of meaningful haptic CMC devices.

Open Access This article is distributed under the terms of the Creative Commons Attribution 4.0 International License (http://creativecomm ons.org/licenses/by/4.0/), which permits unrestricted use, distribution, and reproduction in any medium, provided you give appropriate credit to the original author(s) and the source, provide a link to the Creative Commons license, and indicate if changes were made.

\section{References}

1. Ackerley R, Backlund Wasling H, Liljencrantz J, Olausson H, Johnson RD, Wessberg J (2014) Human C-tactile afferents are tuned to the temperature of a skin-stroking caress. J Neurosci 34(8):2879-2883

2. Anderson N, Glickert R (2016) The descendent. Chapman University film school, USA

3. Aron A, Aron EN, Tudor M, Nelson G (1991) Close relationships as including other in the self. J Pers Soc Psychol 60(2):241-253

4. Asch SE (1946) Forming impressions of personality. J Abnorm Psychol 41:258-290

5. Bailenson JN, Yee N, Brave S, Merget D, Koslow D (2007) Virtual interpersonal touch: expressing and recognizing emotions through haptic devices. Human Computer Interact 22:325-353

6. Bargh JA, Shalev I (2012) The substitutability of physical and social warmth in daily life. Emotion 12(1):154-162

7. Bartholomew K, Horowitz LM (1991) Attachment styles among young adults: a test of a four-category model. J Pers Soc Psychol 61(2):226-244

8. Baumeister RF, Leary MR (1995) The need to belong: desire for interpersonal attachments as a fundamental human motivation. Psychol Bull 117(3):497-529

9. Bergman P, Ho HN, Koizumi A, Tajadura-Jiménez A, Kitagawa N (2015) The pleasant heat? evidence for thermal-emotional implicit associations occurring with semantic and physical thermal stimulation. Cogn Neurosci 6(1):24-30

10. Bickmore TW, Fernando R, Ring L, Schulman D (2010) Empathic touch by relational agents. IEEE Trans Affect Comput 1:60-71

11. Bowlby J (1969) Attachment and loss: attachment, vol 1, 2nd edn. Basic Books, New York

12. Bradley MM, Lang PJ (1994) Measuring emotion: the selfassessment manikin and the semantic differential. J Behav Ther Exp Psychiatry 25(1):49-59

13. Cabibihan JJ, Chauhan S (2017) Physiological responses to affective tele-touch during induced emotional stimuli. IEEE Trans Affect Comput 8(1):108-118 
14. Cacioppo JT, Gardner WL (1999) Emotion. Annu Rev Psychol 50:191-214

15. Cacioppo JT, Patrick W (2008) Loneliness: human nature and the need for social connection. W.W. Norton \& Company, New York

16. Clark MS, Reis HT (1988) Interpersonal processes in close relationships. Annu Rev Psychol 39:609-672

17. Coan JA, Schaefer HS, Davidson RJ (2006) Lending a hand: social regulation of the neural response to threat. Psychol Sci 17(12):1032-1039

18. Cohen J (1992) A power primer. Psychol Bull 112(1):155-159

19. Craig AD, Chen K, Bandy D, Reiman EM (2000) Thermosensory activation of insular cortex. Nat Neurosci 3(2):184-190

20. Daft RL, Lengel RH (1986) Organizational information requirements, media richness and structural design. Manage Sci 32(5):554-571

21. Davydov DM, Zech E, Luminet O (2011) Affective context of sadness and physiological response patterns. J Psychophysiol 25(2):67-80

22. DiSalvo C, Gemperle F, Forlizzi J, Montgomery E (2003) The Hug: an exploration of robotic form for intimate communication. Proc Rom 2003:403-408

23. Dolin DJ, Booth-Butterfield M (1993) Reach out and touch someone: analysis of nonverbal comforting responses. Commun $\mathrm{Q}$ 41:383-393

24. Donnellan MB, Lucas RE, Cesario J (2015) On the Association between Loneliness and Bathing Habits: Nine Replications of Bargh and Shalev (2012) Study 1. Emotion 15(1):109

25. Ebersole CR (2016) Many labs 3: evaluating participant pool quality across the academic semester via replication. J Exp Soc Psychol 67:68-82

26. van Erp JBF, Toet A (2015) Social touch in human-computer interaction. Front Digit Humanit 2(2):1-14

27. Faul F, Erdfelder E, Buchner A, Lang AG (2009) Statistical power analyses using $\mathrm{G}^{*}$ Power 3.1: tests for correlation and regression analyses. Behav Res Methods 41(4):1149-1160

28. Fay AJ, Maner JK (2012) Warmth, spatial proximity, and social attachment: the embodied perception of a social metaphor. J Exp Soc Psychol 48(6):1369-1372

29. Field T (2001) Touch. MIT Press, Cambridge

30. Field T (2010) Touch for socioemotional and physical well-being: a review. Dev Rev 30(4):367-383

31. Fiske ST, Cuddy AJC, Glick P (2007) Universal dimensions of social cognition: warmth and competence. Trends Cogn Sci 11(2):77-83

32. Gallace A, Spence C (2010) The science of interpersonal touch: an overview. Neurosci Biobehav Rev 34(2):246-259

33. Gooch D, Watts L (2010) Communicating social presence through thermal hugs. In: Schmid F, Hesselmann T, Boll S, Cheverst K, Kulik L (eds) Proceedings of First Workshop on Social Interaction in Spatially Separated Environments (SISSI2010), International Society for Presence Research, Copenhagen, pp 11-19

34. Gooch D, Watts LA (2012) YourGloves, hothands and hotmits: devices to hold hands at a distance. In: Proceedings of the 25th annual ACM symposium on user interface software and technology, pp 157-166

35. Guest S (2011) The development and validation of sensory and emotional scales of touch perception. Attention Perception Psychophys 73(2):531-550

36. Haans A, IJsselsteijn WA (2006) Mediated social touch: a review of current research and future directions. Virtual Real 9:149-159

37. Hannan D (2010) Coral sea dreaming: awaken. Plankton Productions, Australia

38. Hertenstein MJ (2002) Touch: its communicative functions in infancy. Hum Dev 45(2):70-94
39. Hong J, Sun Y (2012) Warm it up with love: the effect of physical coldness on liking of romance movies. J Consum Res 39(2):293306

40. Huisman G (2017) Social touch technology: a survey of haptic technology for social touch. IEEE Trans Haptics 10(3):391-408

41. IJzerman H, Gallucci M, Pouw WTJL, Weißgerber SC, van Doesum NJ, Williams KD (2012) Cold-blooded loneliness: social exclusion leads to lower skin temperatures. Acta Psychol (Amst) 140(3):283-288

42. IJzerman H, Heine ECE, Nagel SK, Pronk TM (2017) Modernizing relationship therapy through social thermoregulation theory: evidence, hypotheses, and explorations. Front Psychol 8:635

43. IJzerman H, Hogerzeil LJ (2016) People as penguins: thermoregulation as part of the human essence

44. IJzerman H, Karremans JC, Thomsen L, Schubert TW (2013) Caring for sharing. Soc Psychol (Gott) 44(2):160-166

45. IJzerman H, Semin GR (2009) The thermometer of social relations: mapping social proximity on temperature. Psychol Sci 20(10):1214-1220

46. IJzerman H, Semin GR (2010) Temperature perceptions as a ground for social proximity. J Exp Soc Psychol 46(6):867-873

47. Inagaki TK, Eisenberger NI (2013) Shared neural mechanisms underlying social warmth and physical warmth. Psychol Sci 24:2272-2280

48. Inagaki TK, Irwin MR, Eisenberger NI (2015) Blocking opioids attenuates physical warmth-induced feelings of social connection. Emotion 15(4):494-500

49. Ioannou $\mathrm{S}$ et al (2013) The autonomic signature of guilt in children: a thermal infrared imaging study. PLoS One 8(11):e79,440

50. Janssen JH, Bailenson JN, IJsselsteijn WA, Westerink JHDM (2010) Intimate heartbeats: opportunities for affective communication technology. IEEE Trans Affect Comput 1(2):72-80

51. Jones LA, Berris M (2002) The psychophysics of temperature perception and thermal-interface design. In: Proceedings on HAPTICS 2002 IEEE computer society, , pp 137-142

52. Jones LA, Ho HN (2008) Warm or cool, large or small? the challenge of thermal displays. IEEE Trans Haptics 1(1):53-70

53. Kang Y, Williams LE, Clark MS, Gray JR, Bargh JA (2011) Physical temperature effects on trust behavior: the role of insula. Soc Cogn Affect Neurosci 6(4):507-515

54. Kistler A, Mariauzouls C, von Berlepsch K (1998) Fingertip temperature as an indicator for sympathetic responses. Int J Psychophysiol 29(1):35-41

55. Kolb P (2012) The effects of temperature on service employees' customer orientation: an experimental approach. Ergonomics 55(6):621-635

56. Lakoff G, Johnson M (1999) Philosophy in the flesh: the embodied mind and its challenge to western thought. Basic Books, New York

57. Lang PJ (1995) The emotion probe: studies of motivation and attention. Am Psychol 50(5):372-385

58. Laurenceau JP, Barrett LF, Pietromonaco PR (1998) Intimacy as an interpersonal process: the importance of self-disclosure, partner disclosure, and perceived partner responsiveness in interpersonal exchanges. J Pers Soc Psychol 74(5):1238-1251

59. LeBel EP, Campbell L (2013) Heightened sensitivity to temperature cues in individuals with high anxious attachment: real or elusive phenomenon? Psychol Sci 24(10):2128-2130

60. Lee W, Lim YK (2010) Thermo-message: exploring the potential of heat as a modality of peripheral expression. In: Fitzpatrick G, Hudson S, Edwards K, Rodden T (eds) CHI 10: extended abstracts. ACM Press, Atlanta, GA, pp 4231-4236

61. Lee W, Lim Y (2012) Explorative research on the heat as an expression medium: focused on interpersonal communication. Pers Ubiq Comput 16(8):1039-1049 
62. Lykken DT, Venables PH (1971) Direct measurement of skin conductance: a proposal for standardization. Psychophysiology 8(5):656-672

63. Lynott D, Corker KS, Wortman J, Connell L, Donnellan MB, Lucas RE, O'Brien K (2014) Replication of "experiencing physical warmth promotes interpersonal warmth" by Williams and Bargh (2008). Soc Psychol (Gott) 45(3):216-222

64. Mandryk RL, Inkpen KM, Calvert TW (2006) Using psychophysiological techniques to measure user experience with entertainment technologies. Behav Inf Technol 25(2):141-158

65. Melchert CC (2012) Hechtingsstijl, Depressieve Symptomen \& Impliciete Metingen. Ph.D. thesis, Universiteit van Amsterdam

66. Montagu A (1972) Touching: the human significance of the skin. Harper \& Row Publishers, New York

67. Nie J, Park M (2012) Can you hold my hand? physical warmth in human-robot interaction. In: Proceedings of the human-robot interaction, pp 201-202

68. Oostenveld R, Fries P, Maris E, Schoffelen JM (2011) FieldTrip: open source software for advanced analysis of MEG, EEG, and invasive electrophysiological data. Comput Intell Neurosci 2011:1-9

69. Park E, Lee J (2014) I am a warm robot: the effects of temperature in physical humanrobot interaction. Robotica 32(1):133-142

70. Peeters FPML, Ponds RWHM, Vermeeren MTG (1996) Affectiviteit en zelfbeoordeling van depressie en angst. Tijdschr Psychiatr 38(3):240-250

71. Piferi RL, Kline KA, Younger J, Lawler KA (2000) An alternative approach for achieving cardiovascular baseline: viewing an aquatic video. Int J Psychophysiol 37(2):207-217

72. Raison CL, Hale MW, Williams LE, Wager TD, Lowry CA (2015) Somatic influences on subjective well-being and affective disorders: the convergence of thermosensory and central serotonergic systems. Front Psychol 5:1-22

73. Rantala J, Salminen K, Raisamo R, Surakka V (2013) Touch gestures in communicating emotional intention via vibrotactile stimulation. Int J Hum Comput Stud 71(6):679-690

74. Rimé B, Philippot P, Boca S, Mesquita B (1992) Long-lasting Cognitive and Social Consequences of Emotion: Social Sharing and Rumination. In: W. Stroebe, M. Hewstone (eds.) Eur. Rev. Soc. Psychol., vol. 3, chap. 8, pp. 225-258. Wiley, Chichester, UK

75. Rimm-Kaufman SE, Kagan J (1996) The psychological significance of changes in skin temperature. Motiv Emot 20(1):63-78

76. Salminen K, Surakka V (2013) Cold or hot? how thermal stimuli are related to human emotional system? In: haptic audio interaction design, pp. 20-29

77. Salminen K. et al. (2008) Emotional and behavioral responses to haptic stimulation. In: Proceedings on CHI '08, ACM Press, New York, pp 1555-1562

78. Schilder JD, IJzerman H, Denissen JJA (2014) Physical warmth and perceptual focus: a replication of IJzerman and Semin (2009). PLoS One 9(11):1-3

79. Seebode J, Schleicher R, Wechsung I, Möller S (2013) Investigating the affective impression of tactile feedback on mobile devices. In: Proceedings of the 27th international BCS human computer interaction conference pp 1-10

80. Seifi H, Maclean KE (2013) A first look at individuals' affective ratings of vibrations. In: world haptics conference WHC 2013, pp. $605-610$

81. Sumioka H, Nakae A, Kanai R, Ishiguro H (2013) Huggable communication medium decreases cortisol levels. Sci Rep 3(3034):16

82. Sung EJ, Yoo SS, Hyo Woon Y, Oh SS, Han Y, Park HW (2007) Brain activation related to affective dimension during thermal stimulation in humans: a functional magnetic resonance imaging study. Int J Neurosci 117(7):1011-1027
83. Szymkow A, Chandler J, IJzerman H, Parzuchowski M, Wojciszke B (2013) Warmer hearts, warmer rooms: how positive communal traits increase estimates of ambient temperature. Soc Psychol (Gott) 44(2):167-176

84. Tai K, Zheng X, Narayanan J (2011) Touching a teddy bear mitigates negative effects of social exclusion to increase prosocial behavior. Soc Psychol Personal Sci 2(6):618-626

85. Task Force of the European Society of Cardiology (1996) Heart rate variability: standards of measurement, physiological interpretation, and clinical use. Circulation 93(5):1043-1065

86. Teh JKS, Cheok AD, Peiris RL, Choi Y, Thuong V, Lai S (2008) Huggy Pajama: a mobile parent and child hugging communication system. In: Proceedings of the 7th international conference on Interaction design and children, pp. 250-257

87. Tollmar K, Junestrand S, Torgny O (2000) Virtually living together. a design framework for new communication media. In: Proc. 3rd Conf. Des. Interact. Syst. Process. Pract. methods, Tech., pp. 83-91

88. Tsalamlal MY, Martin JC, Ammi M, Tapus A, Amorim MA (2015) Affective handshake with a humanoid robot: how do participants perceive and combine its facial and haptic expressions? In: Proceedings of the 6th Conference on Affective Computing and Intelligent Interaction, pp. 334-340

89. Tsalamlal MY, Ouarti N, Martin JC, Ammi M (2015) Haptic communication of dimensions of emotions using air jet based tactile stimulation. J Multimodal User Interfaces 9(1):69-77

90. Tsetserukou D, Neviarouskaya A (2010) iFeel_IM!: augmenting emotions during online communication. IEEE Comput Graph Appl 30(5):72-80

91. Turkle S (2011) Alone together: why we expect more from technology and less from each other. Basic Books, New York

92. Trounce K, Macé D, Wajsbrot A (2010) Red balloon. Hurricane Productions, France

93. van Acker BB, Kerselaers K, Pantophlet J (2014) IJzerman H. Homely thermoregulation, how physical coldness makes an advertised house a home

94. van den Troost A, Vermulst AA, Gerris JRM, Matthijs K (2005) The dutch marital satisfaction and communication questionnaire: a validation study. Psychol Belg 45(3):185-206

95. Vess M (2012) Warm thoughts: attachment anxiety and sensitivity to temperature cues. Psychol Sci 23(5):472-474

96. Wada K, Shibata T (2006) Living with seal robots in a care house-evaluations of social and physiological influences. In: Proceedings of 2006 IEEE/RSJ international conference on intelligent robots and systems, pp 4940-4945

97. Watson D, Clark LA, Tellegen A (1988) Development and validation of brief measures of positive and negative affect: the PANAS scales. J Pers Soc Psychol 54(6):1063-1070

98. Willemse CJAM (2015) A warm touch of affect? In: Proceedings on affective computing and intelligent interaction, pp 766-771

99. Willemse CJAM, Heylen DKJ, van Erp JBF (2015) Warmth in affective mediated interaction. In: international conference on affective computing and intelligent interaction, pp. 28-34

100. Willemse CJAM, Munters GM, van Erp JBF, Heylen DKJ (2015) Nakama: A companion for non-verbal affective communication. In: Proceedings 2015 ACM international conference on multimodal interaction, pp 377-378

101. Willemse CJAM, Toet A, van Erp JBF (2017) Affective and behavioral responses to robot-initiated social touch: towards understanding the opportunities and limitations of physical contact in human-robot interaction. Front. ICT 4(May)

102. Williams LE, Bargh JA (2008) Experiencing physical warmth promotes interpersonal warmth. Science 322(5901):606-607

103. Williams LE, Huang JY, Bargh JA (2009) The scaffolded mind: higher mental processes are grounded in early experience of the physical world. Eur J Soc Psychol 39:1257-1267 
104. Wilson G, Dobrev D, Brewster SA (2016) Hot under the collar: mapping thermal feedback to dimensional models of emotion. In: proceedings of the $2016 \mathrm{CHI}$ conference on human factors in computing systems, pp 4838-4849

105. World Medical Association (2013) World medical association declaration of Helsinki: ethical principles for medical research involving human subjects. J Am Med Assoc 310(20):2191-2194

106. Yamazaki R (2016) Intimacy in phone conversations: anxiety reduction for danish seniors with hugvie. Front Psychol 7:1-9

107. Yarosh S, Markopoulos P, Abowd GD (2014) Towards a questionnaire for measuring affective benefits and costs of communication technologies. In: Proceedings of the 17th ACM conference on Computer supported cooperative work \& social computing, pp 84-96
108. Zhong CB, Leonardelli GJ (2008) Cold and lonely: does social exclusion literally feel cold? Psychol Sci 19(9):838-842

Publisher's Note Springer Nature remains neutral with regard to jurisdictional claims in published maps and institutional affiliations. 